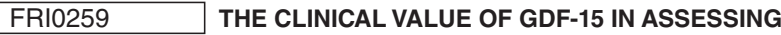 MYOCARDIAL INVOLVEMENT OF IDIOPATHIC INFLAMMATORY MYOPATHY}

M. Qiu' ${ }^{1}$ X. Sun ${ }^{2}$, F. Lu², Q. Wang ${ }^{2}$, L. Zhou ${ }^{3}{ }^{1}$ Nanjing Medical University Graduate School, Nanjing, China; ${ }^{2}$ The First Affiliated Hospital of Nanjing Medical University, Nanjing, China; ${ }^{3}$ Department of Cardiology, The First Affiliated Hospital of Nanjing Medical University, Nanjing, China

Background: Cardiac involvement is a serious complication of idiopathic inflammatory myopathy (IIM). Early diagnosis and intervention can improve prognosis. At present, myocardial biopsy is the gold standard for its diagnosis, but it is not commonly used because of its invasiveness. Biomarkers can be invoked as a non-invasive and convenient choice. The traditional markers of myocardial injury, as troponin and creatine kinase are lack specificity in inflammatory myopathy, so the novel biomarkers are getting attention.GDF-15 can predict the risk of cardiovascular disease and the prognosis of coronary atherosclerosis, heart failure and other diseases. Objectives: This article was intended to investigate the diagnostic value of GDF15 for myocardial involvement in inflammatory myopathy.

Methods: This retrospective study included 54 patients with inflammatory myopathy from May 2018 to October 2019. Of these,30 patients underwent cardiac magnetic resonance examination due to increased myocardial markers, excluding 1 case of severe lung infection. 33 patients with systemic lupus erythematosus (SLE), 16 normal patients were used as the control group. The concentration of GDF-15 in the serum of all groups of patients was measured by ELISA.

Results: 1. There were significantly differences in GDF-15 levels in patients with inflammatory myopathy, systemic lupus erythematosus and normal subjects $(\mathrm{H}=39.870, \mathrm{P}<0.001) .2 .29$ patients with cardiac magnetic resonance on the basis of the delayed enhancement (LGE) and ECV results were divided into two groups in which 19 patients with myocardial injury group and 10 patients without myocardial injury. The best cut-off value was calculated by ROC curve,and comparing GDF-15 and CKMB with the optimum cut-off values in predicting cardiac involvement in IIM.GDF-15 levels were statistically significant between the myocardial injury group $(1765.868 \pm 1068.549 \mathrm{pg} / \mathrm{ml})$ and the group without myocardial injury $(689.967 \pm 458.12 \mathrm{pg} / \mathrm{ml})(\mathrm{p}=0.0011)$. At the same time, the creatine kinase isoenzyme $(\mathrm{CKMB})(158.583 \pm 119.389 \mathrm{U} / \mathrm{L}$ vs $57.96 \pm 52.673 \mathrm{U} / \mathrm{L}, \mathrm{p}=0.005)$ was statistically different between the two groups.3.GDF-15 $\geq 1005.3650 \mathrm{pg} / \mathrm{ml}$ (AUC $=0.853,95 \% \mathrm{Cl} 0.694-1.000$ ) predicted myocardial involvement in inflammatory diseases with a sensitivity of 0.765 and specificity of 0.900 . The AUC of the ROC curve for the joint detection of GDF-15 and CKMB was $0.888,95 \%$ $\mathrm{Cl} 0.757-1.000$, with the predicted probability cut-off value in 0.3895 , the sensitivity 0.941 and the specificity 0.800 . The combined detection of the two increased the sensitivity of myocardial damage detection in IIM patients. 5. After adjusted for age, renal function, the risk of myocardial injury in IIM patients increased by an average of $0.3 \%$ per unit of GDF-15(OR $=1.003,95 \% \mathrm{Cl} 1.000-1.005)$.

Conclusion: GDF-15 can predict myocardial injury in patients with inflammatory myopathy which have high specificity. The prediction sensitivity can be improved by combining with the traditional myocardial enzyme CKMB.More further studies are needed to confirm the specific mechanism of GDF-15 for myocardial involvement to assess the prognosis of such patients and guide further treatment.

References:

[1] Sultan SM, Ioannou Y, Moss K, Isenberg DA. Outcome in patients with idiopathic inffllammatory myositis: morbidity and mortality. Rheumatology (Oxford) 2002;41:22-6.

[2] Lundberg IE, de Visser M, Werth VP. Classification of myositis. Nat Rev Rheumatol. 2018 May; 14(5):269-278.

[3] Zhang L, Wang GC, Ma L, Zu N (2012) Cardiac involvement in adult polymyositis or dermatomyositis: a systematic review. Clin Cardiol 35(11):686-691.

[4] Chen F,Peng Y,Chen M. Diagnostic approach to cardiac involvement in idiopathic inflammatory myopathies.A strategy combining cardiac troponin I but not $T$ assay with other methods[J].Int Heart J,2018;59:256-262

Disclosure of Interests: None declared

DOI: 10.1136/annrheumdis-2020-eular.2841

\section{FRI0260 NAILFOLD VIDEOCAPILLAROSCOPY IS A USEFUL TOOL TO RECOGNIZE SYSTEMIC SCLEROSIS AND IDIOPATHIC INFLAMMATORY MYOPATHIES IN INTERSTITIAL LUNG DISEASE PATIENTS}

G. Sambataro ${ }^{1,2}$, D. Sambataro ${ }^{2}$, F. Pignataro ${ }^{3}$, N. Del Papa ${ }^{3}$, M. Colaci ${ }^{1}$, L. Malatino ${ }^{1}$, A. Libra' ${ }^{1}$, F. Pino ${ }^{1}$, S. E. Torrisi' ${ }^{1}$, S. Palmucci ${ }^{1}$, L. Cavagna ${ }^{4}$, C. Vanchieri'. ${ }^{1}$ University of Catania, Catania, Italy; ${ }^{2}$ Artroreuma S.R.L., Mascalucia (CT), Italy; ${ }^{3}$ ASST G. Pini-CTO, Milan, Italy, Milan, Italy; ${ }^{4}$ University of Pavia, Pavia, Italy

Background: Nailfold Videocapillaroscopy (NVC) is an essential tool for the assessment of Raynaud's Phenomenon (RP) among the Scleroderma Spectrum Disorders (SSDs). Recently, NVC abnormalities have been associated with a diagnosis of Idiopathic Inflammatory Myopathies (IIMs), independently of the presence of RP (1). Moreover, both SSDs and IIMs are commonly associated with Interstitial Lung Disease (ILD), which is the main cause of mortality in these conditions.

Objectives: To verify whether NVC may allow a better diagnostic classification in a cohort of patients with ILD followed by pulmonologists.

Methods: 361 patients affected by ILD were prospectively enrolled in a 30-months observational study. All these patients were clinically evaluated by rheumatologists and pulmonologists together and performed general blood tests, autoantibody research, chest High-Resolution Computed Tomography and NVC The latter was considered positive in the presence of avascular areas or giant capillaries, and also the presence of Bushy Capillaries (BCs) was recorded.

Results: NVC was positive in $17.7 \%$ of ILD patients, and a third of these patients did not present RP. Patients with NVC abnormalities had a diagnosis of definite Connective Tissue Disease in $78.1 \%$ of cases. NVC resulted decisive in $25 \%$ of patients with a final diagnosis of Systemic Sclerosis according to the ACR/ EULAR 2013 criteria. The presence of BCs and/or NVC+ in ILD patients with normal serum levels of muscular enzymes was associated with amyopathic IIM (BCs= OR 3.9, 95Cl 1.05-14.38, $\mathrm{p}=0.04$; NVC+= OR 5, 95Cl 1.29-19.3 $\mathrm{p}=0.02$ $\mathrm{BCs}$ and/or NVC+= OR $5.4195 \mathrm{Cl} 1.24-23.48 \mathrm{p}=0.02$ ), regardless the presence of RP.

Conclusion: NVC proved to be a valid tool in the correct assessment of ILD patients secondary to SSDs and amyopathic IIMs. Therefore, it could be considered in the diagnostic evaluation of patients affected by ILD, regardless of the presence of RP.

\section{References:}

[1] Sebastiani M et al. Nailfold Capillaroscopy Characteristics of Antisynthetase Syndrome and possible clinical associations: results of a multicenter International Study. J Rheumatol 2019; 46: 279-284

Table 1. Comparison between ILD patients with and without NVC positivity.

\begin{tabular}{lccc}
\hline Items & $\begin{array}{c}\text { NVC+ } \\
\text { patients }\end{array}$ & NVC-patients & $\mathbf{p}$ \\
\hline Number & 64 & 297 & \\
Mean Age $( \pm$ SD) & $61.4 \pm 13.3$ & $67.8 \pm 9.8$ & 0.001 \\
Female\% & 65.6 & 49.1 & 0.01 \\
RP\% & 68.7 & 17.1 & $<0.0001$ \\
HRCT patterns\% & & & \\
NSIP & 51.5 & 36.8 & 0.02 \\
OP & 3.1 & 8 & n.s. \\
UIP-like & 31.15 & 44.8 & n.s. \\
DIP & 1.5 & 3.4 & n.s. \\
LIP & 1.5 & 0.4 & n.s. \\
Indeterminate & 10.9 & 6.7 & \\
Final Diagnosis\% & & & $<0.0001$ \\
SSDs & 45.3 & 1 & $<0.0001$ \\
IIMs & 25 & 6.4 & n.s. \\
Other CTDs & 6.2 & 13.5 & n.s. \\
IPAF & 10.9 & 19.5 & 0.0007 \\
IPF & 7.8 & 27.9 & \\
IPF & & &
\end{tabular}

Legend: DIP: Desquamative Interstitial Pneumonia; HRCT: High Resolution Computed Tomography; IIMs: Idiopathic Inflammatory Myopathies, Poly/dermatomyositis, antisynthetase syndrome; IPAF: Interstitial Pneumonia with Autoimmune Features; IPF: Idiopathic Pulmonary Fibrosis; LIP: Lymphocytic Interstitial Pneumonia; NSIP: Nonspecific Interstitial Pneumonia; OP: Organising Pneumonia; RP: Raynaud's Phenomenon; SSDs: Scleroderma Spectrum Disorders, Systemic Sclerosis + Mixed Connective Tissue Disease; UIP: Usual Interstitia Pneumonia.

Disclosure of Interests: Gianluca Sambataro: None declared, Domenico Sambataro: None declared, Francesca Pignataro: None declared, Nicoletta Del Papa None declared, Michele Colaci: None declared, Lorenzo Malatino: None declared, Alessandro Libra: None declared, Fabio Pino: None declared, Sebastiano Emanuele Torrisi Speakers bureau: Boehringer Ingelheim; F. Hoffmann-La Roche Ltd., Stefano Palmucci Consultant of: Boehringer Ingelheim, Speakers bureau: Boehringer Ingelheim, Delphi International Srl, and F. Hoffmann-La Roche Ltd. Lorenzo Cavagna: None declared, Carlo Vanchieri Grant/research support from: F. Hoffmann-La Roche Ltd., Consultant of: AstraZeneca, Boehringer Ingelheim, Chiesi, F. Hoffmann-La Roche Ltd., and Menarini, Speakers bureau: AstraZeneca, Boehringer Ingelheim, Chiesi, F. Hoffmann-La Roche Ltd., and Menarini DOI: 10.1136/annrheumdis-2020-eular.4724

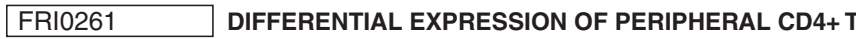 CELLS IN PATIENTS WITH SYSTEMIC SCLEROSIS AND MIXED CONNECTIVE TISSUE DISEASE}

L. Shang ${ }^{1}$, T. Zhang ${ }^{2}$, J. Luo ${ }^{2}$, J. Yuan ${ }^{2}$, C. Gao ${ }^{3}$, X. F. LI ${ }^{4}$, H. Gao ${ }^{2}{ }^{1}$ The Second Hospital of Shanxi Medical University, Department of Rheumatology, Taiyuan, China; ${ }^{2}$ The Second Hospital of Shanxi Medical University, Department of Rheumatology, Taiyuan, China; ${ }^{3}$ Brigham and Women's Hospital, Department 